\title{
Alterstice
}

Revue internationale de la recherche interculturelle

International Journal of Intercultural Research

Revista International de la Investigacion Intercultural

\section{Le théâtre-forum comme outil de recherche et d'intervention par et pour les jeunes issus de l'immigration}

\section{Caterine Bourassa-Dansereau}

Volume 7, numéro 1, 2017

L'interculturel dans la Cité : actes à poser en contexte pluriethnique

URI : https://id.erudit.org/iderudit/1040611ar

DOI : https://doi.org/10.7202/1040611ar

Aller au sommaire du numéro

Éditeur(s)

Alterstice

ISSN

1923-919X (numérique)

Découvrir la revue

Citer cet article

Bourassa-Dansereau, C. (2017). Le théâtre-forum comme outil de recherche et d'intervention par et pour les jeunes issus de l'immigration. Alterstice, 7(1), 51-62. https://doi.org/10.7202/1040611ar

\section{Résumé de l'article}

Nous montrons dans cet article comment le théâtre-forum peut devenir un outil de recherche et d'intervention auprès des jeunes issus de l'immigration. Plus spécifiquement, nous présentons différents « actes à poser " qui répondent aux enjeux liés à la reconnaissance et à l'expression de la complexité identitaire des jeunes en contexte de diversité. Pour ce faire, notre réflexion s'effectue en trois temps : après la présentation sommaire du théâtre-forum, nous montrons premièrement l'importance et les apports de ce type d'intervention en ce qui a trait aux actes posés par les jeunes eux-mêmes et associés à leur prise de parole, au développement d'un regard réflexif et à la conscientisation individuelle et collective. Nous soulignons comment ces actes s'articulent aux particularités de la recherche et de l'intervention auprès des jeunes issus de l'immigration. Dans un deuxième temps, nous illustrons ces actes grâce à une démarche de théâtre participatif particulière, ModÉgalité, dans laquelle le théâtre-forum a été utilisé comme outil principal d'intervention. Cette recherche-action a été menée auprès de jeunes adultes montréalais de 16 à 22 ans issus de l'immigration et portait sur leurs perceptions de l'égalité entre les femmes et les hommes. Ces exemples concrets nous permettent non seulement d'illustrer les actes posés par ces jeunes mais aussi d'aborder les actes à poser par les intervenants dans ce contexte. Nous soumettons finalement au lecteur nos réflexions concernant les défis liés à ces actes d'intervention lors de la mise en place d'une démarche mobilisant le théâtre-forum, notamment auprès des jeunes issus de l'immigration. En définitive, cet article met en lumière comment les « actes à poser » associés au théâtre-forum amènent à ne plus penser, parler et agir au nom des jeunes issus de l'immigration, mais plutôt par et pour ceux-ci.
Ce document est protégé par la loi sur le droit d'auteur. L’utilisation des services d’Érudit (y compris la reproduction) est assujettie à sa politique d'utilisation que vous pouvez consulter en ligne.

https://apropos.erudit.org/fr/usagers/politique-dutilisation/ 


\section{7}

ARTICLE THÉMATIQUE

\section{Le théâtre-forum comme outil de recherche et d'intervention par et pour les jeunes issus de l'immigration}

Caterine Bourassa-Dansereau ${ }^{1}$

\section{Résumé}

Nous montrons dans cet article comment le théâtre-forum peut devenir un outil de recherche et d'intervention auprès des jeunes issus de l'immigration. Plus spécifiquement, nous présentons différents " actes à poser » qui répondent aux enjeux liés à la reconnaissance et à l'expression de la complexité identitaire des jeunes en contexte de diversité. Pour ce faire, notre réflexion s'effectue en trois temps : après la présentation sommaire du théâtreforum, nous montrons premièrement l'importance et les apports de ce type d'intervention en ce qui a trait aux actes posés par les jeunes eux-mêmes et associés à leur prise de parole, au développement d'un regard réflexif et à la conscientisation individuelle et collective. Nous soulignons comment ces actes s'articulent aux particularités de la recherche et de l'intervention auprès des jeunes issus de l'immigration. Dans un deuxième temps, nous illustrons ces actes grâce à une démarche de théâtre participatif particulière, ModÉgalité, dans laquelle le théâtre-forum a été utilisé comme outil principal d'intervention. Cette recherche-action a été menée auprès de jeunes adultes montréalais de 16 à 22 ans issus de l'immigration et portait sur leurs perceptions de l'égalité entre les femmes et les hommes. Ces exemples concrets nous permettent non seulement d'illustrer les actes posés par ces jeunes mais aussi d'aborder les actes à poser par les intervenants dans ce contexte. Nous soumettons finalement au lecteur nos réflexions concernant les défis liés à ces actes d'intervention lors de la mise en place d'une démarche mobilisant le théâtre-forum, notamment auprès des jeunes issus de l'immigration. En définitive, cet article met en lumière comment les " actes à poser » associés au théâtre-forum amènent à ne plus penser, parler et agir au nom des jeunes issus de l'immigration, mais plutôt par et pour ceux-ci.

\section{Rattachement de l'auteure}

${ }^{1}$ Université du Québec à Montréal (UQAM), Montréal, Canada

\section{Correspondance}

bourassa-dansereau.caterine@uqam.ca

\section{Mots clés}

théâtre-participatif; immigration; jeunesse; recherche; intervention

\section{Pour citer cet article}

Bourassa-Dansereau, C. (2017). Le théâtre-forum comme outil de recherche et d'intervention par et pour les jeunes issus de l'immigration. Alterstice, 7(1), 51-62. 


\section{Mise en contexte : quelle voix pour les jeunes issus de l'immigration ?}

Les jeunes issus de l'immigration ${ }^{1}$ sont porteurs de plusieurs référents identitaires, notamment ceux associés à leurs appartenances ethnoculturelles ${ }^{2}$ et à leur âge, qui peuvent être liés à leur exclusion de l'espace public en tant que citoyens actifs et reconnus. Les jeunes issus de l'immigration sont en effet " socialement invisibles " (Honneth, 2004) : il y a peu de reconnaissance de leurs expériences et de leurs savoirs. Différents auteurs ont montré à ce propos qu'être jeunes, immigrants ou membres de communautés ethnoculturelles minoritaires peut être lié à diverses situations de discrimination et que la voix des individus porteurs de ces appartenances est socialement peu sollicitée et surtout, rarement entendue (Gallant et Pilote, 2013, Jacquet et Masinda, 2014; Potvin et Lafortune, 2014).

Paradoxalement, les actes posés en contexte de diversité, notamment les différents protocoles de recherche et d'intervention "interculturels" s'adressant à la population "jeunes issus de l'immigration " peuvent parfois favoriser cette exclusion sociale, en étiquetant ces individus comme étant d'abord et avant tout issus de la diversité. En effet, les jeunes participants peuvent y être essentiellement définis en fonction de leurs appartenances ethnoculturelles, sous le regard d'intervenants et de chercheurs qui entament l'interaction avec un individu qu'ils considèrent d'abord - voire uniquement - en fonction des référents associés à son statut " issu de l'immigration » (Bourassa-Dansereau, 2014). Le processus d'assignation identitaire (Lypianski, 1993; Maalouf, 1998) qui en découle pourra alors favoriser l'érection d'appartenances ethnoculturelles en identité, conséquence d'un processus qu'entretiennent et alimentent souvent malgré eux les chercheurs et intervenants (Buittelar, 2006; Gallant, 2013).

Dans le cadre de cet article, nous montrons comment le théâtre-forum peut être considéré comme un outil de recherche et d'intervention auprès de ces jeunes en répondant aux préoccupations liées à la reconnaissance et à l'expression de leur complexité identitaire. Quelques recherches ont déjà montré la pertinence de différentes approches mobilisant le théâtre participatif ${ }^{3}$, notamment auprès de populations stigmatisées (McAll, 2012) et de populations jeunes et issues de l'immigration (Ghebaur, 2014; Guilbert, 2004; Wendell, 2014). De notre côté, nous articulons la réflexion présentée dans cet article autour du théâtre-forum et de trois objectifs principaux. Suite à une présentation sommaire du théâtre-forum, nous montrons d'abord l'importance et les apports de cette approche pour la prise de parole, le développement d'un regard réflexif et d'une conscientisation individuelle et collective. Nous soulignons de plus comment ces éléments répondent aux particularités de la recherche et de l'intervention auprès des jeunes issus de l'immigration. Dans un deuxième temps, nous illustrons nos réflexions à travers une démarche de théâtre participatif précise, celle du projet ModÉgalité et de la pièce de théâtre-forum qui y est associée. Cette illustration met en lumière comment les différents actes associés au théâtre-forum nous amènent à ne plus penser, parler et agir au nom des jeunes issus de l'immigration, mais plutôt par et pour ceux-ci. Enfin, nous soumettons au lecteur nos réflexions concernant les défis liés à la mise en place d’une démarche de recherche et d'intervention mobilisant le théâtre-forum, notamment auprès de cette population.

\section{Le théâtre-forum : éléments de définition}

Le théâtre-forum s'inscrit directement dans le Théâtre de l'opprimé, un théâtre à la fois " esthétique et politique ", développé dans les années 1970 au Brésil par Augusto Boal (1977). Celui-ci affiliait cette approche à la Pédagogie des opprimés de Paulo Freire (1974), mettant de l'avant l'idée que l'utilisation du « dialogue comme espace transitif d'apprentissage [est] propice aux changements sociaux » (Hamel, 2012, p. 10).

\footnotetext{
1 Par «jeunes issus de l'immigration", nous entendons les jeunes de première génération d'immigration (jeunes nés à l'étranger et ayant immigré au Canada) et de deuxième génération d'immigration (jeunes nés au Canada de parents nés à l'étranger).

${ }^{2}$ L'appartenance ethnoculturelle renvoie aux dimensions ethniques (liées au sentiment d'appartenance individuelle à un groupe culturel donné) et aux référents culturels (langue, région géographique d’origine, pratique religieuse, etc.) (Côté, 2012).

${ }^{3}$ Différentes approches font appel au théâtre participatif pour intervenir auprès de groupes et de communautés ; le théâtreforum (Boal, 1977), le théâtre social (Wendell, 2014), le théâtre d'action (Brahy, 2014), le théâtre de l'opprimé (Lénel, 2011) et le théâtre engagé ou d'intervention (Biot, Wibo et Ingberg, 2000; Ducharme, 2009) en sont des exemples.
} 
On peut définir le théâtre-forum comme étant un outil de changement et de mobilisation qui permet aux membres d'un groupe minoritaire ou vulnérable ${ }^{4}$ de revisiter des situations préoccupantes ou problématiques vécues, de multiplier les réflexions à leur sujet afin d'en faire une analyse distanciée et, ultimement, de les transformer. Le théâtre-forum se distingue ainsi du théâtre conventionnel par sa volonté d'intervention et de « mise en action » du public (Boal, 1977 et 1978), notamment en impliquant directement ce dernier dans les différentes étapes de la création théâtrale. Le théâtre-forum amène de plus les spectateurs à interagir directement auprès des personnages de la pièce, les faisant ainsi passer du rôle traditionnellement plus passif de spectateur à celui plus actif de spectacteur (Biot, 2000; Ducharme, 2009).

Comme l'explique Boal $(1977 ; 1978)$, afin de permettre la " mise en action » du public lors du théâtre-forum, le groupe à qui l'on s'adresse est d'abord consulté afin de définir quels sont ses préoccupations, difficultés et questionnements face à la problématique ou situation sur laquelle on souhaite intervenir. Par la suite et à partir des propos recueillis, les animateurs-acteurs ${ }^{5}$ proposent une pièce (scénarisée ou improvisée) illustrant une ou plusieurs des réalités exposées par ce public. Cette production théâtrale doit représenter le plus fidèlement possible les perceptions et expériences partagées par le groupe, utiliser son langage (les mots et expressions employés dans la pièce doivent être à l'image de ceux qu'utilisent les membres du groupe) et se terminer sur une " solution insatisfaisante " ou une "impasse ". Suite à la présentation de la pièce, on réalise ensuite la partie "forum » : les animateurs-acteurs ouvrent le débat avec le public et demandent aux participants leurs réactions, réflexions et pistes de solution concernant la situation théâtralisée. Les différents points de vue, compréhensions et analyses sont alors exprimés et entendus. Les animateurs-acteurs rejouent ensuite la pièce, ou certaines de ses parties, mais cette fois en intégrant les apports du public (concernant par exemple de meilleures façons de "faire " ou de " dire " pour les personnages) et même en invitant certains spect-acteurs à monter sur scène afin de prendre leur place et ainsi, "jouer» leurs propres solutions. À travers la mise en action propre au théâtre-forum, on propose donc au public une situation théâtrale correspondant à leur réalité sur laquelle ils doivent agir et qu'il leur faut « repenser et transformer » (Lénel, 2011, p. 93).

L'objectif du théâtre-forum est donc de permettre la conscientisation et d'encourager l'action, et ce, sur les plans individuel et collectif. II s'agit en effet, selon Boal (1977, p. 256), de « [...] transformer le spectateur en protagoniste d'action théâtrale et, par cette transformation, de tenter de modifier la société et non de se contenter de l'interpréter ". Dans cet article, nous traitons de trois actes spécifiques que posent les jeunes participant au théâtre-forum et qui sous-tendent cet objectif principal : la prise de parole, le développement et l'énonciation d'un regard réflexif et la mise en perspective des dimensions collectives des expériences individuelles théâtralisées. Nous montrons de plus comment chacun de ces aspects peut répondre aux exigences des " actes à poser " lors de recherches et interventions menées auprès de jeunes issus de l'immigration.

\section{Prendre la parole : l'acte au coeur du théâtre-forum}

Nous présentons ici l'importance et les implications de la prise de parole lors du théâtre-forum. À travers cet acte posé par les jeunes, il s'agit de faciliter, favoriser et valoriser l'expression et la reconnaissance des voix de personnes habituellement absentes ou exclues des espaces publics et des discours dominants. Selon Boal (1977, p. 263), la prise de parole peut prendre différentes formes lors du forum et "le débat, le conflit d'idées, la dialectique, l'argumentation, la contre-argumentation, tout cela stimule, éveille, enrichit, prépare le spectateur à agir dans la vie réelle ". Comme dans toutes démarches de théâtre participatif, l'acte à poser est donc

\footnotetext{
${ }^{4}$ Le théâtre de l'opprimé est à l'origine une réponse aux différentes répressions qui s'exerçaient en Amérique latine au cours des années 1970 (Boal, 1977, 1978; Lénel, 2011). Cette approche, résolument politique, s'est aujourd'hui quelque peu transformée, passant d'un théâtre participatif axé presque exclusivement sur l'oppression liée aux « luttes de classes » dans les années 70 et 80 (par exemple concernant la condition des ouvriers) à un théâtre participatif intervenant auprès de groupes " opprimés " ou "exclus " en raison de leurs appartenances à différents groupes minoritaires (personnes souffrant d'un handicap, issues de l'immigration, en situation socio-économique précaire, etc.), notamment dans une optique d'accompagnement et d'insertion (Brahy, 2014).

${ }^{5}$ Nous utilisons le terme animateurs-acteurs pour désigner les intervenants qui assument à la fois le rôle d'acteur lors de la présentation de la pièce et d'animateur lors de la partie forum et au fil des interactions avec le public.
} 
premièrement celui de la prise de parole. La discussion et le dialogue ont ainsi préséance sur la recherche de résultats ou de solutions concrètes aux problématiques exposées dans la pièce (Boal, 1977; Brahy, 2014).

Cette prise de parole n'est pas anodine. En effet, elle attribue aux personnes du public la position d'expert de leur propre vie : leurs savoirs expérientiels, ainsi que leurs connaissances de la problématique liée à leurs expériences quotidiennes et personnelles sont sollicités et reconnus. Ce sont les principaux acteurs " dans la vraie vie » de ces situations théâtralisées et ils détiennent, par le fait même, le savoir qui sera non seulement requis et entendu lors du théâtre-forum, mais aussi mis en valeur et cautionné à travers les situations théâtrales "transformées " qui résultent de leurs discussions, propositions et pistes de solution. Dans ce processus de co-construction qui prend place au cœur des interactions de groupe, s'établit alors un dialogue ouvert caractérisé par des rapports de pouvoir qui se veulent horizontaux et par la reconnaissance des savoirs et contributions mutuelles (Freire, 1974). Comme dans tout processus de co-construction, cet espace de prise de parole vise, dans une symétrie relationnelle, à favoriser l'autonomie de chacun, le respect et la confiance réciproque (Lafranchise, 2016).

Pour les jeunes issus de l'immigration, souvent socialement disqualifiés, la prise de parole qui caractérise le théâtre-forum et la reconnaissance des savoirs et des expériences qu'elle induit sont essentielles. Le processus entourant l'acte de prendre parole (et son corollaire, l'acte de la recevoir) permet aux individus de développer, à travers les interactions qui caractérisent le théâtre-forum, des savoirs individuels et collectifs positifs et valorisés.

\section{La théâtralisation de l'expérience : acte réflexif du théâtre-forum}

Différents auteurs ont montré que les connaissances se construisent à partir de l'expérience et grâce au regard réflexif que les individus posent sur leurs propres actions et expériences (Argyris et Shön, 1974; Bourassa, Serre et Ross, 1999; Kolb, 1984). Pour Lafortune (2011, p. 3), " Une pratique réflexive suppose une mise à distance et un regard critique sur son propre fonctionnement, mais aussi une analyse tant individuelle que collective des actions et décisions prises en cours d'action ». Au cours du théâtre-forum, c'est essentiellement grâce à l'acte de distanciation que permet la théâtralisation des situations vécues par le public que le regard réflexif se déploie en cours de démarche. En effet, "la projection par le jeu des remplacements de personnages permet une distanciation favorisant la prise en compte de sa propre réalité » (Hamel, 2012, p. 73) et la réflexion critique qui s'ensuit se co-construit au cours des discussions entre les individus et au sein du groupe. En assistant à la mise en scène de situations qu'ils ont vécues, puis en les ré-interprétant à travers les discussions et en montant eux-mêmes sur scène pour modifier la trame narrative, les individus sont amenés à analyser et nommer leurs expériences et à agir sur elles. Ainsi, ces distanciations permettent le développement et l'énonciation du regard réflexif porté sur les expériences et perceptions. En effet, les discussions collectives qui accompagnent la théâtralisation s'arriment alors aux réflexions individuelles et favorisent, dans ce mouvement réflexif et itératif, l'évolution de certaines compréhensions ou analyses initiales. Ce mouvement mène aussi le spect-acteur à l'action : en agissant sur l'action dramatique, il le fait par extension sur sa vie (Boal, 1977).

Dans un contexte de diversité culturelle, cette pratique réflexive est à souligner. Elle favorise chez les jeunes une réflexion sur leurs propres pratiques du "vivre ensemble » et une vision nuancée de leur rapport à l'altérité. C'est ce que constate Wendell (2014, p. 94) dans une étude portant sur les apports du théâtre social dans la mobilisation des élèves en tant que " citoyens créateurs" et qui, selon l'auteur, " [...] s'avère un bon moyen de reconnaître la diversité existant dans la société ainsi que d'accroître chez l'élève une vision enrichie et approfondie. ». Wendell poursuit en ajoutant que " le processus de création favorise l'apprentissage du vivre ensemble, dans une société diversifiée qui se caractérise par de multiples références. » (2014, p. 94).

\section{Du « je » au " nous » : ancrage social des expériences individuelles}

Nous souhaitons également aborder les actes associés à la conscientisation individuelle et collective qui ponctuent le théâtre-forum. C'est notamment lors des périodes de forum et des discussions collectives qui les caractérisent que chaque individu est amené à resituer ses expériences individuelles à l'intérieur de structures et rapports sociaux plus larges dans lesquelles elles s'insèrent et qui les définissent (Boal, 1977, 1978; Hamel, 2012; Lénel, 2011; Wendell, 2014). Les discussions autour des situations théâtralisées et des expériences et réflexions de chaque participant permettent alors aux individus de constater que l'expérience des autres fait écho à la sienne. 
Ainsi, le théâtre-forum met en lumière le rapport social qui sous-tend les expériences individuelles ponctuelles ou quotidiennes, et permet au participant de "[...] passer du " je » au "chacun ", de faire apparaître le commun " (Lénel, 2011, p. 101). La situation ou problématique théâtralisée n'est donc plus seulement appréhendée sous son angle singulier et occasionnel, elle l'est aussi dans sa dimension structurelle et systémique. En définitive, le théâtreforum permet la prise de conscience des rapports de pouvoir qui sous-tendent les relations interpersonnelles (Hamel, 2012).

Lorsqu'il est question de jeunes issus de l'immigration, ce passage de l'individuel au collectif nous semble particulièrement pertinent. En effet, les différentes situations de discrimination pouvant être vécues au quotidien par ces jeunes se doivent d'être appréhendées au-delà des seules dimensions individuelles ou interpersonnelles. L'acte à poser est alors celui qui permet, à travers le théâtre-forum, de reconnaître que ces situations s'inscrivent dans des structures et rapports sociaux précis propres à différents groupes d'appartenance (famille, religion, langue, communauté d'origine, etc.). L'acte favorise alors, chez les jeunes adultes, l'énonciation, la dénonciation, voire la transformation de ces situations.

\section{Projet ModÉgalité : actes posés par les jeunes issus de l'immigration}

Pour illustrer la pertinence du théâtre-forum comme " acte à poser » auprès de jeunes issus de l'immigration, nous présentons le projet ModÉgalité (Bourassa-Dansereau, 2013). Cette recherche-action a été menée entre 2011 et 2014 grâce à la collaboration de trois organismes montréalais : le Forum jeunesse de l'île de Montréal, le Y des femmes de Montréal et l'organisme de théâtre participatif Mise au jeu ${ }^{6}$. Nous avons agi à titre de chercheure pour la réalisation de ce projet qui visait à documenter les perceptions et expériences de jeunes adultes montréalais de 16 à 22 ans concernant l'égalité entre les femmes et les hommes. Spécifions que cette recherche a été menée à la fois auprès de jeunes adultes issus de l'immigration et de jeunes non issus de l'immigration ${ }^{7}$. Pour les besoins de cet article, nous considérons uniquement la démarche, les données et les résultats obtenus auprès des jeunes adultes issus de l'immigration ${ }^{8}$.

\section{ModÉgalité : une démarche de théâtre participatif}

Pour le projet ModÉgalité, les jeunes adultes issus de l'immigration ont d'abord été rencontrés lors de groupes de discussion où étaient proposés aux participants, outre des discussions, différents exercices théâtraux. Grâce à ces rencontres, nous avons pu accéder aux perceptions, préoccupations et expériences de ces jeunes concernant l'égalité entre les femmes et les hommes. À travers des échanges construits autour des exercices théâtraux, ces derniers ont non seulement pris la parole sur ce sujet, mais se sont aussi " mis en scène " à travers différentes situations d'(in)égalité entre hommes et femmes qui les interpellaient dans les sphères publique (milieux scolaires, professionnels, univers médiatiques) et privée (expériences familiales et relations amoureuses).

À l'aide des propos recueillis lors de ces rencontres, la pièce de théâtre-forum ModÉgalité a été créée par l'équipe de théâtre participatif Mise au jeu. Cette pièce met en scène des situations d'égalité et d'inégalité vécues par les jeunes, telles qu'ils nous les ont présentées lors des groupes de discussion. Elle a été créée à partir des propos recueillis et les thèmes suivants, articulés aux appartenances ethnoculturelles, y sont principalement abordés : les perceptions de la nature égalitaire ou complémentaire des relations entre les hommes et femmes, les rôles sexuels qui caractérisent les relations amoureuses, les expériences et les représentations associées à la sexualité féminine

\footnotetext{
${ }^{6}$ Le Projet ModÉgalité a été rendu possible grâce au soutien financier du Secrétariat à la condition féminine du Québec.

${ }^{7}$ Nous spécifions que les résultats obtenus auprès des deux groupes sont similaires en ce qui a trait aux grands thèmes abordés, soit la nature égalitaire ou complémentaire des relations de couple, les rôles sexuels, la sexualité et les influences sociales et médiatiques. Les groupes composés de jeunes issus de l'immigration ont toutefois fortement mobilisé leurs appartenances ethnoculturelles pour aborder ces questions, contrairement aux jeunes non issus de l'immigration.

${ }^{8}$ Les données et résultats utilisés pour cet article ont été recueillis lors de cinq groupes de discussion menés auprès de jeunes issus de l'immigration (groupes non-mixtes au niveau du genre et composés en fonction des groupes d'âge, 16-18 ans et 1922 ans) et dans le cadre de quatre théâtres-forum réalisés dans des milieux scolaires et communautaires caractérisés par leur diversité ethnoculturelle (les théâtres-forum ont réuni des participants masculins et féminins, en fonction des mêmes groupes d'âge).
} 
et masculine, les modèles masculins et féminins dans les médias et, finalement, la préoccupation pour une égalité sociale, au-delà d'une égalité de genre et entre les sexes. La pièce de théâtre-forum ModÉgalité a été présentée à l'automne 2011 aux jeunes participants et ces derniers ont été invités à partager leurs interprétations et analyses à son propos, en plus d'être conviés, lors du forum, à monter sur scène pour donner la réplique aux personnages et proposer des solutions alternatives à certaines situations.

Dans les sections suivantes, nous montrons comment ModÉgalité a permis aux jeunes issus de l'immigration de poser les actes associés à la prise de parole et au développement d'un regard réflexif et d'une analyse à la fois individuelle et collective de situations d'(in)égalité. Nous abordons aussi les actes posés par les intervenants afin de favoriser la mise en action des jeunes participants.

\section{Prendre la parole, être entendu et reconnu : la voix des jeunes issus de l'immigration}

La revue de littérature réalisée en amont du projet ModÉgalité nous avait permis de mettre en lumière le constat suivant: la parole des jeunes adultes montréalais issus de l'immigration sur la question de l'égalité entre les femmes et les hommes était peu sollicitée et entendue (Bourassa-Dansereau, 2013). À ce moment, différents auteurs (Beaudoin, Lepage et Bérubé, 2007; Descarries et Mathieu, 2009; Plamondon, Desaulniers et Roy, 2008; Riberdy, Lavoie et Fournier, 2007; Surprenant, 2009) s'étaient en effet intéressés à des aspects spécifiques de l'égalité au Québec (au sein de la famille, du couple, au travail, etc.), mais seules quelques études abordaient la question de façon générale chez les jeunes (Farrand, Deshaies, Harvey et Mailloux, 2009) ou en prenant en compte l'appartenance ethnoculturelle (Beaulieu, Adrien et Lebounga Vouma, 2011; Chouakri, 2009; Guilbault et Di Dominico, 2005). De plus, la littérature ne permettait pas toujours d'accéder aux perceptions et aux expériences de ces jeunes adultes sur cette question. La recherche-action ModÉgalité visait donc à donner la parole aux jeunes adultes sur ces questions et à les amener, à travers une démarche de théâtre participatif, à partager et à faire valoir leurs savoirs expérientiels à ce propos.

Dans le cadre du processus participatif et pour atteindre cet objectif de prise de parole, certains actes étaient à poser par les intervenants. Nous avons dans un premier temps tenté de limiter l'impact des rapports de pouvoir perçus entre les jeunes participants et les animateurs-acteurs, les chercheurs et les intervenants. La démarche devait en effet mettre en place des conditions relationnelles permettant l'émergence de dialogues authentiques. En ce sens, nous avons notamment mené la démarche avec des animateurs-acteurs recrutés non seulement en fonction de leurs compétences et expertises théâtrales, mais aussi parce qu'ils partageaient certains référents identitaires avec les membres des groupes rencontrés: ils étaient jeunes (tous âgés de moins de 30 ans) et majoritairement issus de l'immigration. Ces caractéristiques personnelles ont permis de limiter les incidences du rapport de pouvoir perçu entre les « adultes-intervenants » et les " jeunes-participants » et surtout, la formulation de "bonnes " réponses, c'est-à-dire celles perçues par les jeunes comme étant jugées "acceptables " par les différents intervenants. Les animateurs-acteurs ont par ailleurs précisé aux participants, dès le début des rencontres, qu'aucun propos ne serait jugé et que tous étaient libres d'exprimer ce qu'ils pensaient et ressentaient, sans retenue. Les propos suivants d'un jeune participant ${ }^{9}$ illustrent l'importance d'instaurer les conditions optimales pour l'émergence d'une parole authentique, ainsi que les dangers d'autocensure que l'on peut associer à toute démarche participative :

J'espère que vous n'allez pas être choqués par ce qu'on va vous dire là. Vous voulez qu'on parle de filles et de nous et de nos relations. Je ne sais pas, aussi de sexe? Est-ce qu'on peut dire tout ce qu'on pense vraiment ou bien est-ce qu'il faut qu'on parle correct? (GD, H., 16-18 ans)

Dans le cadre du projet ModÉgalité, l'acte associé à la mise en place d'un espace perçu comme étant dénudé de jugement ou d'apriori visait à favoriser l'expression des représentations et expériences d'(in)égalité vécues par les

\footnotetext{
${ }^{9}$ Tous les extraits présentés sont issus de verbatim réalisés suite à la tenue des groupes de discussion et des représentations de théâtre-forum. Pour en faciliter la lecture, nous avons retranscrit les propos recueillis pour qu'ils correspondent à un langage écrit et avons corrigé certaines erreurs de syntaxe et de vocabulaire en prenant soin de ne pas altérer le sens des témoignages. Tous les extraits seront identifiés en fonction du type de collecte de données (groupe de discussion, GD ou théâtre-forum, TF), du genre du participant (homme, H. ou femme, F.) et de son groupe d'âge (16-18 ans ou 19-22 ans).
} 
jeunes. C'est par ailleurs à travers différentes formes de dialogue allant de l'approbation au débat enflammé, entre les jeunes eux-mêmes et avec les animateurs-acteurs, que ces derniers ont pu faire entendre et reconnaître leurs voix à ce propos.

Les jeunes issus de l'immigration ont utilisé cet espace de parole pour exposer la richesse et la variété de leurs expériences et perceptions concernant l'égalité entre les femmes et les hommes. Ils ont aussi pu y exprimer, de leur propre chef, certaines de leurs particularités en associant largement leurs réflexions à leurs appartenances ethnoculturelles. Par exemple, les jeunes ont abordé ce qu'ils perçoivent comme l'influence de leur communauté d'origine sur la nature (in)égalitaire de leurs relations amoureuses, sexuelles ou familiales. Les extraits suivants illustrent leurs réflexions à ce propos :

Chez les Haïtiens, 80 \% sont divorcés. C'est rare qu'ils sont en couple et qu'ils ont une belle relation. C'est parce que lorsqu'ils se marient, les maris sont souvent infidèles et donc, ça finit par un divorce. Les relations de couple sont difficiles parce que les femmes haïtiennes sont des femmes fortes et que culturellement, elles ont appris à ne pas se laisser faire. Alors les hommes et les femmes haïtiens, dans leur relation, c'est comme de l'huile et du feu! (TF, H., 16-18 ans)

Si une fille a ce genre de relations [sexuelles] là, elle va vraiment se faire traiter de noms. Mais pour le gars, c'est complètement le contraire! Pour eux on se dit : c'est normal, nos hommes dans notre culture, ils ont le sang chaud. (TF, F., 19-22 ans)

Mon frère est plus jeune que moi, mais il est plus libre que moi. Avec lui, tout se discute, mais moi, je dois obéir à mes parents et surtout à mon père. C'est vraiment une grosse influence culturelle cette façon-là de voir la famille. C'est parce que c'est la façon de faire pour élever les enfants dans notre pays. (GD, F., 16-18 ans)

À l'image de leur identité complexe composée de référents identitaires hétérogènes, la démarche a aussi permis aux participants d'affirmer et d'expliciter la pluralité de leurs représentations concernant l'égalité entre les femmes et les hommes. En effet, ces derniers ont souligné que leurs perceptions et expériences ne se réduisent pas à leurs appartenances ethnoculturelles et que d'autres appartenances participent à la construction de celles-ci. À ce sujet, certains jeunes ont dénoncé le fait qu'on leur attribue parfois des « façons de faire ou de voir » l'égalité, en raison de leur statut « issu de l'immigration ». Aussi, plusieurs jeunes adultes ont partagé leur satisfaction de pouvoir faire entendre leurs perceptions de l'égalité dans le cadre du projet ModÉgalité. Les propos suivants témoignent à la fois de leur vision plurielle de l'égalité, de la complexité de leur identité et de l'importance accordée à un espace de parole qui reconnaît leurs expériences et savoirs sur la question de l'égalité :

Ce qui est frustrant pour moi, c'est que comme je suis voilée, les gens pensent tout de suite que je vais être soumise dans mon couple ou que plus tard, je vais rester à la maison et pas travailler [...]. Mais ce n'est pas toujours ça. J'aime ça pouvoir le dire que non, ce n'est pas juste ça. (TF, F., 16-18 ans)

C'est sûr que quand je pense à ça [l'égalité], c'est comme un mélange. Je ne suis pas né ici, mais je pense un peu plus comme les Québécois maintenant. Parce que je suis né ailleurs, j'ai mon bagage culturel, oui, mais j'habite ici maintenant et je ne suis plus vraiment le même qu'à mon arrivée. Je vois ça différemment. Le problème, c'est que des fois, c'est les autres qui pensent que les immigrants ne peuvent pas avoir changé. Et ce n'est pas toujours facile pour moi de dire autre chose. (GD, H., 16-18 ans)

À travers la mise en place d'un espace de parole, le projet ModÉgalité a donc permis aux jeunes issus de l'immigration de partager leur lecture de l'égalité entre les femmes et les hommes en fonction de leur réalité. La démarche a favorisé leur prise de parole et celle-ci a été reçue et entendue à travers les différentes étapes du projet. Pour ces jeunes participants "socialement invisibles» (Honneth, 2004), l'acte de parole favorise l'expression et permet la reconnaissance d'une identité individuelle et collective riche et complexe. Comme en témoigne ce participant lors du théâtre-forum présenté à son école secondaire, il permet surtout d'exprimer les expériences et perceptions associées à une réalité définie par les jeunes eux-mêmes, en dehors des normes de discours institutionnels et majoritaires :

Je pense que les profs dans la salle ont eu les cheveux qui se sont levés sur la tête [rire]. [Ce qu'on a dit] Ce n'est pas vraiment ce qu'on va dire habituellement à l'école. Ce n'est pas comme " ce qu'il faut penser à l'école " qu'on a dit 
aujourd'hui et pour moi, c'est bien qu'on puisse dire ce que nous les Haïtiens on pense de ça. Parce que ce n'est pas vraiment pareil comme ce qu'on entend d'habitude quand on parle du couple des Québécois. Ou bien quand les Québécois parlent de nous [rire]. (TF, H., 19-22 ans)

\section{Le regard réflexif : de l'individuel au collectif}

Le théâtre-forum propose aux spect-acteurs la théâtralisation de situations vécues afin qu'ils réfléchissent de façons individuelle et collective à leur sujet et qu'ils développent un regard réflexif critique permettant la conscientisation individuelle et collective menant à l'action (Boal, 1977, 1978).

Les actes ayant ponctué le processus du projet ModÉgalité ont favorisé le déploiement de ce regard réflexif chez les jeunes participants issus de l'immigration. La mise à distance provoquée par la théâtralisation de situations d'(in)égalité a permis aux jeunes participants de préciser, de nuancer et parfois même de remettre en question certaines de leurs perceptions ou pratiques. Ce regard réflexif s'est développé grâce aux mouvements itératifs entre le "théâtre » et la "vraie vie » que propose le théâtre-forum et à travers les interactions entre les jeunes participants. La réflexion individuelle et collective émergeant du théâtre-forum a par ailleurs permis aux participants de resituer les situations individuelles présentées dans la pièce à l'intérieur de structures et rapports sociaux plus larges.

Nous illustrons ces constats à l'aide d'un exemple spécifique : celui des perceptions de l'égalité entre les femmes et les hommes en matière de sexualité. Lors des groupes de discussion, les questions liées à l'égalité et à la sexualité ont en effet occupé une grande partie des discussions, notamment dans les groupes composés de jeunes hommes. Les participants ont entre autres partagé une représentation selon laquelle certaines jeunes femmes « utilisent le sexe " pour " manipuler " les hommes et " obtenir ce qu'elles veulent » de leur partenaire. Suite à ces affirmations et afin d'engager un processus réflexif au sein du groupe de discussion, les animateurs-acteurs invitent les jeunes à explorer cette représentation en y posant un "deuxième regard ", à travers un exercice théâtral qui les amène à mettre en scène une situation illustrant leurs propos. Deux jeunes hommes se prêtent alors au jeu et proposent, de façon spontanée, la scène suivante au groupe: il s'agit de la représentation d'un acte sexuel où un homme est debout et une femme, devant lui, est à genoux. Les animateurs demandent ensuite aux jeunes participants de "faire parler" les personnages afin que le groupe puisse accéder à ce qu'ils " pensent ». Étonnamment, loin d'exprimer une intention liée à la manipulation ou à l'instrumentalisation de la sexualité, le participant incarnant le personnage féminin dira alors : "j'aimerais ça avoir du plaisir moi aussi, il me semble que c'est juste toi qui en as ». Le personnage masculin répondra alors : « oui, mais le sexe, c'est pour les hommes ». S'ensuivra une discussion où seront (re)discutées les représentations associées à la sexualité féminine et masculine et où différentes nuances et précisions seront apportées par les jeunes à ce niveau. À travers les actes des animateurs-acteurs, ceux des jeunes et l'action théâtrale, on assiste alors à une certaine transformation de la représentation qu'illustrent les propos suivants d'un participant " c'est sûr que je dis encore qu'il y a [des filles qui] utilisent le sexe pour avoir ce qu'elles veulent, mais je pense que c'est aussi parce que c'est souvent le seul choix que leur laisse la société ». Cet exemple montre comment les différents actes des intervenants (proposer la "mise en scène " de leurs représentations et engager la discussion à leur propos) amènent les jeunes à poser, lors et à travers l'action théâtrale, un deuxième regard sur leurs perceptions et expériences et comment ce "retour sur soi » permet le développement d'une analyse réflexive critique. Dans la discussion suivant cet exercice théâtral, les jeunes hommes précisèrent et nuancèrent en effet leurs propos initiaux, notamment en abordant la question de la satisfaction sexuelle des femmes et des hommes au sein du couple. Dans une diversité d'opinions et de positions allant de la " responsabilité des femmes à revendiquer leurs droits » en la matière à la prise de position pour " l'importance du plaisir féminin ", les jeunes nuancèrent leurs perceptions construites à la fois dans une analyse individuelle et collective.

Après les groupes de discussion, cette réflexion critique s'est poursuivie lors de la présentation du théâtre-forum, où le public fut amené à réfléchir, nuancer et discuter ces mêmes enjeux autour de la sexualité féminine. La dimension collective de leurs réflexions et le passage du je au nous se sont alors fortement exprimés, les jeunes participants inscrivant leurs analyses à ce propos à la fois dans les rapports sociaux liés au genre et ceux liés aux appartenances ethnoculturelles. Lors du forum et grâce aux échos que chaque expérience individuelle trouve dans celles des autres, les jeunes adultes ont ainsi fait ce passage réflexif de l'individuel au collectif, notamment en 
abordant la question du double-standard en matière de sexualité qu'ils inscrivent dans des enjeux sociaux. Au-delà des aspects individuels associés à leur propre expérience, ils mettent alors en lumière les structures qui contraignent et orientent les actions et perceptions individuelles. Les propos suivants recueillis lors des théâtresforum illustrent les prises de conscience à la fois individuelle et collective dont il est question :

Moi, je me reconnais dans [le personnage de] la fille. On voit que si la fille veut vivre des relations [sexuelles], elle va être traitée de pute. Et que si c'est un gars, là, c'est vraiment cool. C'est ça qu'elle veut faire arrêter. (TF, F., 16-18 ans)

Dans la pièce, la fille parle de son droit de vivre comme elle veut et je suis d'accord. Mais elle ne parle pas de comment va réagir sa famille. Et je pense que c'est quand même important. Elle dit « moi je m’assume, je sais ce que je vaux et ce que les autres pensent je m'en fous ". C'est bien. Mais ce n'est pas si facile, avec ses parents et ce que vont penser les voisins et même toute la communauté. Je le sais que ce n'est pas évident, surtout si on est une fille. (TF, F., 16-18 ans)

Grâce au regard réflexif qui caractérise le processus itératif du théâtre-forum, les jeunes sont amenés à (ré)examiner leurs perceptions et actions de façon critique, à travers leurs propres analyses et celles qu'ils construisent au cœur de l'interaction avec les autres. Cet aspect collectif de la réflexion les amène de plus à reconnaître le caractère commun et partagé de leurs expériences individuelles et à les resituer dans les rapports sociaux où elles prennent place. Du côté des intervenants, c'est donc en en amenant les jeunes à prendre la parole puis à interagir autour d'une pièce créée à partir de leurs propres expériences que le théâtre-forum devient « l'acte à poser » qui favorise la conscientisation individuelle et collective dans une approche où l'on ne fait pas au nom des jeunes, mais bien par et pour ceux-ci.

\section{Théâtre-forum : questionnements et défis des actes d'intervention}

Le projet de recherche-action ModÉgalité permet de souligner les apports du théâtre-forum dans le cadre d'une démarche de recherche ou d'intervention auprès de jeunes issus de l'immigration. Nous souhaitons toutefois soulever certains questionnements et défis associés à la mise en place de cette approche et aux actes d'intervention qui lui sont associés.

Nous voulons souligner premièrement les défis associés à l'animation participative lors du théâtre-forum. Les pièces de théâtre-forum sont souvent composées de stéréotypes et de scènes à caractère caricatural qui visent à susciter la réflexion et la discussion au sein du public concerné. Or une animation inadéquate de la partie "forum ", où la déconstruction des stéréotypes et caricatures doit être menée avec le public, pourrait au contraire cristalliser ou renforcer certains de ceux-ci. Comme le mentionne Boal (1978, p. 256), il faut s'assurer lors du forum de laisser au public la possibilité d'agir et de réfléchir sur les situations opprimantes ou discriminatoires présentées et " ouvrir des brèches de libération et non acculer les gens à la résignation ". Par exemple, une pièce théâtralisant sans nuance une situation inégalitaire au sein d'un couple « issu de l'immigration » devra être discutée, analysée et déconstruite lors du forum afin de permettre la réflexion critique et constructive sur ces situations problématiques. Dans le cas contraire, et si l'animation participative est mal menée, la pièce risque de n'ajouter qu'une pierre de plus à l'édifice de la discrimination en nourrissant les stéréotypes et préjugés. Ainsi, l'acte d'intervention à poser est d'envergure : il devra permettre l'instauration d'un espace de discussion qui favorise chez le participant le " passage à l'acte ", celui qui permet la prise de parole, le développement et l'énonciation du regard réflexif et la conscientisation individuelle et collective. On peut à ce sujet se questionner sur les conditions souvent précaires de plusieurs milieux d'intervention, qui limitent peut-être la portée, voire rendent impossible pour les professionnels d'instaurer les conditions nécessaires au bon déroulement des théâtres-forum.

Si le théâtre-forum doit permettre aux jeunes issus de l'immigration de prendre la parole, celle-ci doit de plus être entendue et reconnue par les chercheurs et intervenants qui coordonnent la démarche. À ce propos, différents projets de théâtre participatif moins heureux ont parfois failli à cet objectif, soit en n'arrivant pas à représenter les expériences vécues par le groupe en raison d'un langage théâtral non significatif pour celui-ci (Boal, 1977), soit en réactivant, à travers l'exercice participatif, les relations de pouvoir qu'elles voulaient pourtant dénoncer (Ghebaur, 2014; Hamel, 2012). En effet, un des défis du théâtre-forum pour les intervenants est l'acte associé à la reconnaissance de l'expérience et des savoirs des participants. Les participants doivent y être reconnus comme des êtres à la fois uniques et universels, c'est à dire comme des individus singuliers (à travers la spécificité de leurs 
expériences) et universellement reconnus (comme ayant la même valeur que toute autre personne) (Ricœur, 2004). Sans cette reconnaissance, les spect-acteurs se retrouvent discrédités au cœur même de l'expérience participative, où leurs paroles et savoirs entrent en concurrence avec celle des experts universitaires ou professionnels. L'expérience, " au lieu de susciter une prise de conscience [...] apparaît alors, au contraire, comme porteuse d'un discours et d'une volonté de domination, d'écrasement, ainsi que d'un rapport problématique à l'autre. » (Ghebaur, 2014, p. 112). Ainsi, l'acte à poser, par les intervenants, ne peut se faire sans prendre en compte la relation de pouvoir qui définit nécessairement la relation entre les participants et les intervenants. Dans l'approche participative où l'on cherche justement à faire par et pour les jeunes issus de l'immigration, l'acte à poser est aussi celui qui reconnaît la présence de ces relations de pouvoir et tente de les atténuer (notamment en énonçant clairement aux participants que l'espace de parole du théâtre-forum se veut dénudé de jugements et d'aprioris).

Finalement, il nous semble important de mentionner que le théâtre-forum vise, au-delà de la conscientisation, une mise en action et une transformation sociale (Boal, 1977, 1978). Si, comme l'illustre le projet ModÉgalité, le changement individuel et même groupal nous semble atteignable, il apparaît toutefois utopiste de penser que cette approche se traduise par de réels changements au niveau sociétal. En ce sens, la portée du théâtre-forum est avant tout au niveau individuel, éventuellement interpersonnel et groupal. L'acte du théâtre-forum amène ainsi surtout une conscientisation individuelle concernant certaines situations spécifiques. Dans le cas de ModÉgalité, il apparaît en effet que la prise de conscience de chaque participant se déploie d'abord et avant tout au niveau de ses propres relations.

\section{Conclusion}

Cet article nous a permis de montrer comment le théâtre-forum peut devenir un outil de recherche et d'intervention auprès des jeunes adultes issus de l'immigration en répondant aux préoccupations liées à la reconnaissance de ce qu'ils sont et à l'expression de leurs savoirs, de leurs expériences et de leur complexité identitaire. Nous avons montré qu'il permet aux jeunes qui y participent de prendre la parole, de développer un regard réflexif et critique sur leurs représentations et pratiques et d'inscrire l'analyse de leurs expériences dans un cadre non seulement individuel mais aussi collectif. À travers le projet ModÉgalité, nous avons ainsi montré que le théâtre-forum se veut un " acte à poser " qui permet de ne plus penser, parler et agir au nom des jeunes issus de l'immigration, mais plutôt par et pour ceux-ci.

Dans notre contribution, nous nous sommes essentiellement concentrée sur l'acte à poser par les jeunes euxmêmes. Dans un avenir rapproché, il nous semblerait pertinent d'explorer plus en détail la piste de "l'acte à poser " par les intervenants du théâtre-forum : quel sens donnent-ils à leurs actions et interventions dans le cadre de celui-ci? Comment inscrivent-ils ces actes dans les relations de pouvoir qui caractérisent toute relation entre intervenants et participants? Comment les articulent-ils à la diversité (ethnoculturelle, économique, de genre, etc.)? En guise de conclusion, laissons à ce propos la parole à un animateur-acteur du projet ModÉgalité qui terminait une rencontre avec de jeunes participants avec ces mots: "Merci de votre participation et de votre honnêteté. À certains moments, vous nous avez secoués et on a dû, nous aussi, réfléchir à ce qu'on pensait être l'égalité. Et même, remettre en question certaines de nos idées! »

\section{Références bibliographiques}

Argyris, C. et Schön, D. A. (1974). Theory in practice: increasing professional effectiveness. San Francisco : JosseyBass Publishers.

Beaudoin, P., Lepage, F. et Bérubé, F. (2007). Maintenir le cap sur l'égalité de fait : réflexion sur certains enjeux en matière de politique familiale. Québec : Conseil du statut de la femme. http://site.ebrary.com/id/10264470

Beaulieu, M., Adrien, A. et Lebounga Vouma, J. (2011). Facteurs de risque aux infections transmissibles sexuellement et par le sang chez les jeunes Québécois d’origine haïtienne étude exploratoire. Montréal : Direction de santé publique, Agence des services sociaux de Montréal. http://site.ebrary.com/id/10465084 
Biot, P., Wibo, A. et Ingberg, H. (2000). Le théâtre d'intervention aujourd'hui. Louvain-la-Neuve (Belgique) : Centre d'études théâtrales.

Boal, A. (1977). Théâtre de l'opprimé. Paris : La Découverte.

Boal, A. (1978). Jeux pour acteurs et non-acteurs pratique du Théâtre de l'opprimé. Paris : La Découverte.

Bourassa, B., Serre, F. et Ross, D. (1999). Apprendre de son expérience. Sainte-Foy, Québec : Presses de l'Université du Québec.

Bourassa-Dansereau, C. (2013). ModÉgalité : Les perceptions des jeunes Montréalaises et Montréalais sur l'égalité entre les femmes et les hommes. Montréal : Forum jeunesse de l'île de Montréal. http://fjim.org/v3/modegalite.pdf

Bourassa-Dansereau, C. (2014). Les représentations de l'égalité entre les femmes et les hommes de jeunes montréalais issus de l'immigration : illustrations et réflexions à travers le prisme de la communication interculturelle. Commposite, 17(1), 28-52. http://www.commposite.org/index.php/revue/article/viewFile/187/156

Brahy, R. (2014). L'engagement en présence : I'atelier de théâtre-action comme support à une participation sociale et politique ?, Lien social et Politiques, 71,31-39. doi : 10.7202/1024737ar

Buitelaar, M. (2006). «I Am the Ultimate Challenge »: Accounts of Intersectionality in the Life-Story of a WellKnown Daughter of Moroccan Migrant Workers in the Netherlands. European Journal of Women's Studies, 13(3), 259-276.

Chouakri, J. (2009). Femmes immigrantes nouvellement arrivées et égalité entre les sexes : État de la situation dans la région de Montréal. Montréal : Table des groupes de femmes de Montréal.

Côté, D. (2012). La notion d'appartenance ethnoculturelle dans la recherche et l'intervention en réadaptation. Montréal, Québec : IRSST. http://site.ebrary.com/id/10863647

Descarries, F. et Mathieu, M. (2009). Entre le rose et le bleu : stéréotypes sexuels et construction sociale du féminin et du masculin. Québec : Conseil du statut de la femme.

Ducharme, F. (2009). Distinguer le théâtre d'intervention du théâtre engagé. Actes du colloque Engagement : imaginaires et pratiques, Postures, 109-126. oic.uqam.ca/sites/oic.uqam.ca/files/oic1/ducharme-distinguerle-theatre.pdf

Farand, B., Deshaies, M.-H., Harvey, H. et Mailloux, T. (2009). Regards de jeunes sur l'égalité : la perception des jeunes de 15 à 25 ans. Québec : Conseil du Statut de la femme.

Freire, P. (1974). Pédagogie des opprimes. Paris : Maspero.

Gallant, N. (2013). Regard sur... le rôle du regard d'autrui dans la construction identitaire des jeunes. Dans Gallant, N. et Pilote, A. (dir.), La construction identitaire des jeunes (p. 212-230). Québec : Presses de l'Université Laval.

Gallant, N. et Pilote, A. (2013). La construction identitaire des jeunes. Québec: Presses de l'Université Laval.

Ghebaur, C. (2014). "C'est pas pour nous normalement » : La médiation culturelle et les non-publics. Tumultes, 42(1), 101-112. doi : 10.3917/tumu.042.0101

Guilbault, D. et Di Dominico, M. (2005). Des nouvelles d’elles : les femmes immigrées du Québec. Québec : Conseil du statut de la femme.

Guilbert, L. (dir.). (2004). Médiations et francophonie interculturelle. Québec : Les Presses de l’Université Laval.

Hamel, S. (2012). Le Théâtre-forum : Pour une délibération sous le signe de la reconnaissance? (Thèse de doctorat inédite, Université de Montréal). Université de Montréal.

Honneth, A. (2004). Visibilité et invisibilité. Sur l'épistémologie de la « reconnaissance ». Revue du MAUSS, 23(1), 137-151. doi : 10.3917/rdm.023.0137 
Jacquet, M. et Masinda, M. T. (2014). Réflexions sur la notion d'intégration scolaire des jeunes immigrants. International Journal of Canadian Studies, 50, 277-296. doi :10.3138/ijcs.2014.015

Kolb, D. A. (1984). Experiential learning: experience as the source of learning and development. Englewood Cliffs : Prentice-Hall.

Lafortune, L. (2011). Pratique réflexive et dimension affective : réflexion et analyse de ses pratiques comportant une mise à distance critique associée à la compréhension des réactions affectives. Sciences croisées, (7-8), 1-15.

Lafranchise, N. (2016). Co-construction des connaissances à propos des groupes de co-développement professionnel : une recherche-action collaborative et partenariale. Communication présentée dans le cadre du $84^{\mathrm{e}}$ congrès de l'ACFAS, Montréal, Canada.

Lénel, P. (2011). Théâtre de l'opprimé et intervention sociale : Aux sources de l'éducation populaire ? Agora débats/jeunesses, 58(2), 89-104. doi : 10.3917/agora.058.0089

Lipiansky, E.-M. (1993). L'identité dans la communication. Communication et langages, 97(1), 31-37. doi : 10.3406/colan.1993.2452

Maalouf, A. (1998). Les identités meurtrières. Paris : Grasset.

McAll, C. (2012). Au-delà du préjugé : trajectoires de vie, pauvreté, et santé. Québec : Presses de l'Université du Québec.

Plamondon, G., Desaulniers, A. et Roy, N. (2008). Le sexe dans les médias : obstacle aux rapports égalitaires. Québec : Conseil du statut de la femme.

Potvin, M. et Lafortune, G. (2014). Les jeunes d'origine haïtienne au Québec, d'hier à aujourd'hui. Diversité urbaine, 14(2), 3-17. doi : 10.7202/1035422ar

Riberdy, H., Lavoie, S. et Fournier, M. (2007). Enquête sur le bien-être des jeunes montréalais : rapport thématique. Montréal : Agence de la santé et des services sociaux de Montréal. http://www4.banq.qc.ca/pgq/2007/3323097.pdf

Ricœur, P. (2004). Parcours de la reconnaissance : trois études. Paris : Stock.

Surprenant, M.-È. (2009). Jeunes couples en quête d'égalité. Montréal : Sisyphe.

Wendell, N. (2014). L'enseignement du théâtre social à l'école : réflexions sur la mobilisation des élèves en tant que citoyens créateurs. L'Annuaire théâtral : Revue québécoise d'études théâtrales, 55, 89-100. doi : 10.7202/1033704ar

Alterstice - Revue Internationale de la Recherche Interculturelle, vol. 7, $n^{\circ} 1$ 